\title{
Unusual Cryptosporidium species recovered from human faeces: first description of Cryptosporidium felis and Cryptosporidium 'dog type' from patients in England
}

\author{
S. PEDRAZA-DíAZ, C. AMAR, A. M. IVERSEN*, P. J. STANLEY ${ }^{\dagger}$ and J. McLAUCHLIN
}

Food Safety Microbiology Laboratory, Division of Gastrointestinal Infections, PHLS Central Public Health Laboratory, 61 Colindale Avenue, London NW9 5HT, * East Sussex, Brighton and Hove Health Authority, 36-38 Friars Walk, Lewes, East Sussex BN7 2PB and †'Department of Infectious Diseases, Seacroft Hospital, York Road, Leeds LS14 6UH

\begin{abstract}
DNA was extracted from faecal samples collected from 1680 patients in which Cryptosporidium oocysts were recognised by light microscopy. DNA from faeces from five of these patients failed to amplify by PCR three gene fragments - the Cryptosporidium oocyst wall protein (COWP) gene, the thrombospondin-related adhesive protein of Cryptosporidium-1 (TRAP-C1) gene and the thrombospondin-related adhesive protein of Cryptosporidium-2 (TRAP-C2) gene - with primers designed from $C$. parvum sequences. However, DNA from these five patients did amplify cryptosporidial 18S rDNA gene fragments and a heat-shock protein (HSP70) gene fragment was also amplified from four of them. The purpose of this study was to characterise further the Cryptosporidium associated with infection in these patients. DNA sequence analysis of $18 \mathrm{~S}$ rDNA genes showed that four of these patients were infected by $C$. felis, and the remaining one by an as yet un-named Cryptosporidium species designated the 'dog type' $(C$. dt). Infection by $C$. felis was further confirmed in all four patients by DNA sequence analysis of the HSP70 gene. Oocysts present in all five samples reacted strongly with two anti-cryptosporidial oocyst monoclonal antibodies, except for the $C$. $\mathrm{dt}$, which was tested with only one of the antibodies. Two of the patients infected by $C$. felis had underlying illness; one 8-year-old male had an undefined severe inherited underlying condition, and the second patient, a 32-year-old male, was HIV positive. Two of the remaining three patients (two females aged 1 and 2 years, respectively) were apparently immunocompetent (one infected with $C$. felis and one with the $C$. dt). No information was obtained for the fifth patient. The patient infected by $C$. dt had a recent history of travel to Africa. This is the first report of infection with these two Cryptosporidium species in immunocompetent patients, and in any patient in the UK.
\end{abstract}

\section{Introduction}

Cryptosporidia are species of protozoan parasites that infect various mammals, birds, reptiles and fish [1]. Traditionally, the different species of this genus have been identified on the basis of host range, oocyst morphology and site of infection. However, DNA sequence analysis shows the genus to be complex; it may contain $>20$ different species, which cannot be distinguished reliably by traditional methods [1].

Received 23 Aug. 2000; accepted 15 Sept. 2000. Corresponding author: Dr J. McLauchlin (e-mail: jmclauchlin@phls.nhs.uk).
Molecular techniques show Cryptosporidium parvum to be the major cause of cryptosporidiosis in humans and to comprise two different genotypes [1] that may constitute separate species [2]. However, other Cryptosporidium species have also been associated with human infections: C. felis, C. meleagridis and an as yet un-named Cryptosporidium species designated the dogtype $(C . \mathrm{dt})$ have been detected in the faeces of HIVpositive individuals, $[3,4]$. C. meleagridis has also been found in the faeces of immunocompetent individuals [5].

The development of methods for rapid extraction of cryptosporidial DNA from whole faeces [2] and of a nested PCR/RFLP genotyping procedure based upon 
the Cryptosporidium oocyst wall protein (COWP) gene [6] have been described previously. These techniques were applied to faecal specimens from 1680 patients, and the COWP gene fragment was amplified from $95.2 \%$ of the samples [6]. Specimens from some patients failed to amplify the COWP gene fragment, but did amplify fragments of the $18 \mathrm{~S}$ cryptosporidial rDNA gene, and it was suggested that these may represent species of Cryptosporidium other than $C$. parvum [6]. The purpose of the present study was to characterise these cryptosporidia further and to give brief clinical information on the infected patients.

\section{Materials and methods}

The faecal specimens collected were from diarrhoeal patients in which Cryptosporidium oocysts were detected by hospital laboratories by conventional microscopy [7]. Brief clinical and epidemiological details were obtained from the original request forms submitted with the specimen, and further information was obtained by interview of the patient (or where appropriate the patient's parents) with the use of a standard questionnaire.

Specimens were stored at $4^{\circ} \mathrm{C}$ without preservatives and re-examined by light microscopy in the PHLS Food Safety Microbiology Laboratory by modified Ziehl-Neelsen (MZN) acid-fast and immunofluorescence staining [2]. Immunofluorescence staining was performed with two monoclonal antibodies (MAbs) against Cryptosporidium oocyst cell surface: one produced 'in-house' (designated MAB-C1 [2]) and one produced commercially (Cellabs, Brookvale, Australia). Oocyst disruption and DNA purification were performed as described previously [2].

PCR amplification of the 18S rDNA fragment $1[8,9]$, 18S rDNA fragment 2 [2, 10], COWP $[6,11]$, TRAPC1 [12], TRAP-C2 [13] and HSP70 [14] gene fragments were performed as described previously and were analysed by electrophoresis in agarose $1 \%$ / ethidium bromide gels and recorded by UV transillumination with type 667 film (Polaroid, St Albans).
For DNA sequencing, PCR products were purified in Microspin S-400 HR (Pharmacia Biotech, St Albans) and cloned with the TOPO-TA Cloning kit (Invitrogen). Plasmid DNA was purified with the Promega Wizard SV kit (Promega) and clones were sequenced on an ABI377 automated sequencer with BigDye ${ }^{\mathrm{TM}}$ terminator chemistry with the M13 set of primers at the Single Reaction DNA Sequencing Service (Cambridge Bioscience, Cambridge, UK). Sequences were analysed with the Genetics Computer Group (GCG) program package (University of Wisconsin, WI, USA).

\section{Results}

DNA was extracted from faecal samples obtained from 1680 patients and shown to contain cryptosporidial oocysts. Extracts from the faeces of five patients (designated A-E) failed to amplify the COWP, TRAPC1 or TRAP-C2 gene fragments, but did amplify $18 \mathrm{~S}$ rDNA gene fragments 1 and 2, except for the DNA from patient $\mathrm{C}$, which did not amplify fragment 2 . The $18 \mathrm{~S}$ rDNA fragment 1 was sequenced from all five patients (AF323563-AF323567) and found to be identical to the sequence for C. felis (AF087577) in patients A-D, and for the $C$. dt (AF087576) in patient E. Amplification of the HSP70 gene fragment was also achieved from patients A-D. Sequence analysis showed that these fragments (AF323568-AF323571) were identical to the sequence for C. felis (AF221538), confirming the results obtained for the 18S rDNA fragment 1. Examination of the faecal samples from the five patients by conventional light microscopy showed that the oocysts from all five samples were morphologically indistinguishable from $C$. parvum by MZN and initially reacted strongly by immunofluorescence with MAB-C1. All samples were tested by immunofluorescence with the Cellabs MAb at least 1 year later, and all were strongly positive except for the C. dt (patient E), with which no oocysts were detected by either the MAB-C1 or the Cellabs antibodies.

Brief clinical details were obtained for all patients, and further information about two of the cases was obtained by questionnaire (Table 1). Patients B (C. felis) and E $(C . \mathrm{dt})$ were apparently immunocompetent, and no

Table 1. Brief clinical details of patients infected by $C$. felis and the Cryptosporidium dog type

\begin{tabular}{|c|c|c|c|c|c|}
\hline $\begin{array}{l}\text { Patient } \\
\text { number }\end{array}$ & Age & Sex & $\begin{array}{l}\text { Underlying } \\
\text { illness }\end{array}$ & $\begin{array}{l}\text { Date of } \\
\text { collection }\end{array}$ & $\begin{array}{l}\text { Recent travel } \\
\text { history }\end{array}$ \\
\hline \multicolumn{6}{|l|}{ C. felis } \\
\hline A & 8 & M & Yes* & Sept. 1998 & NK \\
\hline B & 2 & $\mathrm{~F}$ & No & Dec. 1998 & No \\
\hline $\mathrm{C}$ & 32 & M & HIV positive & Dec. 1998 & NK \\
\hline $\mathrm{D}$ & 19 & $\mathrm{~F}$ & NK & Sept. 1999 & NK \\
\hline \multicolumn{6}{|c|}{$\begin{array}{l}\text { Cryptosporidium } \\
\text { dog type }\end{array}$} \\
\hline $\mathrm{E}$ & 1 & $\mathrm{~F}$ & No & April 1999 & Africa \\
\hline
\end{tabular}

NK, not known.

*Undefined severe inherited underlying condition. 
other pathogen was identified in their faeces. Patient A had an undefined severe inherited underlying condition, and patient $\mathrm{C}$ was HIV positive. Apart from a recent history of foreign travel with swimming pool use in Africa (patient E), no other risk factors were identified, including exposure to animals or consumption of contaminated drinking water, food or beverages.

\section{Discussion}

Previous studies have shown C. parvum to be the major species of Cryptosporidium in human infection [1]. However, the identification of additional species that affect man [3,4] is important and highlights the need for further molecular studies to enhance understanding of the disease. To achieve this, further characterisation of a wider range of cryptosporidia is needed, together with a re-evaluation of the taxonomy of the genus. Phylogenetic analysis based on DNA sequencing of $18 \mathrm{~S}$ rDNA and a $70-\mathrm{kDa}$ heat-shock protein (HSP70) support the characterisation of $C$. felis and the $C$. dt as distinct from $C$. parvum and as separate species $[1,14,15]$. Both $C$. felis and the $C$. dt have been described in the faeces of HIV-positive individuals $[3,4]$ and this current report is the first to describe infection of immunocompetent patients and of patients in the UK with these two Cryptosporidium species.

As cryptosporidia do not multiply in the environment, and infection results from either direct or indirect exposure to an infected animal [16], an understanding of the host range of different Cryptosporidium species is essential for the identification of reservoirs of infection and ultimately control of the disease. To date, $C$. felis has been isolated from the faeces of cats, a cow and human patients $[3,4,9,17,18]$, and $C$. dt has been recovered from dogs and human patients $[3,19]$. However, because of the similarities in the physical appearance of oocysts produced by different species of Cryptosporidium (see below) and the, as yet, limited application of molecular analysis to larger series of samples from a wide range of potential hosts, the true distribution of these parasites remains to be established.

The reasons for the inability to amplify fragments of the COWP TRAP-C1 and TRAP-C2 genes with the existing primers reported here is not known. Difficulties in amplification of the COWP gene from $C$. meleagridis DNA were most likely to be due to a single base mismatch in one of the primer sequences and were recently reported from this laboratory [5]. A similar explanation is most likely for the PCR amplification failures in the present study. However, this study demonstrates that a combined analysis with PCR procedures for COWP and 18S rDNA gene fragments will identify unusual Cryptosporidium species in human faeces and will require further characterisation by DNA sequencing for identification.
The lack of reaction between the $C$. dt and Cellabs $\mathrm{MAb}$ may be due to spontaneous degradation of the oocysts, as oocysts could not be detected on re-testing the sample with the antibody MAB-C1 c. 1 year after the initial tests. This sample behaved differently from C. parvum oocysts similarly stored in whole faeces, which retained morphology and strong reaction with both the MAbs over much longer periods of time (unpublished data).

The identification of different species of Cryptosporidium in environmental samples relies on the recognition of oocyst morphology in several different staining procedures, including reactions with MAbs [20]. The reaction of these antibodies with $C$. felis and the $C$. dt is reported here, and it has been shown previously that both these antibodies react with $C$. parvum (genotypes 1 and 2), $C$. meleagridis and $C$. wrairi [2, 5, unpublished data]. These five different Cryptosporidium species all produce oocysts of similar size [1] and hence it is not possible to distinguish them reliably by immunofluorescence microscopy. As these species are detected in human infections in very different proportions (see below), there are considerable problems with assessing the public health importance of oocyst detection in water (together with foods or beverages) based solely on the current detection methods using microscopy.

Previous studies have shown that $C$. parvum is the major species involved in human infection [16]. This study, together with other data from this laboratory from $>2000$ cases of cryptosporidiosis, allows an estimation, for the first time, of the distribution of different Cryptosporidium species that cause human disease in the UK $[2,5,6,21$, unpublished data]. Approximately $97 \%$ of cases in which Cryptosporidium oocysts are detected in human faeces are due to $C$. parvum, $1 \%$ to $C$. meleagridis, $0.2 \%$ to $C$. felis and $<0.1 \%$ to the $C$. dt: the remainder have not yet been characterised. This distribution varies markedly in immunocompromised patients where a greater proportion of non-C. parvum species and both $C$. parvum genotypes 1 and 2 occur together (unpublished data). This study, together with other recent advances in the molecular analysis of cryptosporidia, highlights the hitherto uncharacterised complexity of human cryptosporidiosis.

We thank colleagues in clinical microbiology laboratories for the donation of specimens. S.P-D. is funded by Biomed Grant PL 962557 from the European Commission and C.A. is funded by a PHLS PhD studentship.

\section{References}

1. Morgan UM, Xiao L, Fayer R, Lal AA, Thompson RCA. Variation in Cryptosporidium: towards a taxonomic revision of the genus. Int J Parasitol 1999; 29: 1733-1751.

2. McLauchlin J, Pedraza-Díaz S, Amar-Hoetzeneder C, Nichols GL. Genetic characterization of Cryptosporidium strains from 
218 patients with diarrhea diagnosed as having sporadic cryptosporidiosis. J Clin Microbiol 1999; 37: 3153-3158.

3. Pieniazek NJ, Bornay-Llinares FJ, Slemenda SB et al. New Cryptosporidium genotypes in HIV-infected persons. Emerg Infect Dis 1999; 5: 444-449.

4. Morgan U, Weber R, Xiao L et al. Molecular characterization of Cryptosporidium isolates obtained from human immunodeficiency virus-infected individuals living in Switzerland, Kenya, and the United States. J Clin Microbiol 2000; 38: 1180-1183.

5. Pedraza-Díaz S, Amar C, McLauchlin J. The identification and characterisation of an unusual genotype of Cryptosporidium from human faeces as Cryptosporidium meleagridis. FEMS Microbiol Lett 2000; 189: 189-194.

6. Pedraza-Díaz S, Amar C, Nichols GL, McLauchlin J. The development of a nested PCR procedure for amplification of the Cryptosporidium oocyst wall protein (COWP) gene, and analysis of 2128 cryptosporidiosis cases. Emerg Infect Dis 2001 (in press)

7. Arrowood MJ. Diagnosis. In: Fayer R (ed) Cryptosporidium and cryptosporidiosis. Boca Raton, FL, CRC Press. 1997: 4364.

8. Johnson DW, Pieniazek NJ, Griffin DW, Misener L, Rose JB. Development of a PCR protocol for sensitive detection of Cryptosporidium oocysts in water samples. Appl Environ Microbiol 1995; 61: 3849-3855.

9. Bornay-Llinares FJ, da Silva AJ, Moura INS et al. Identification of Cryptosporidium felis in a cow by morphologic and molecular methods. Appl Environ Microbiol 1999; 65: 14551458.

10. Patel S, Pedraza-Díaz S, McLauchlin J. The identification of Cryptosporidium species and Cryptosporidium parvum directly from whole faeces by analysis of a multiplex PCR of the $18 \mathrm{~S}$ rRNA gene and by PCR/RFLP of the Cryptosporidium outer wall protein (COWP) gene. Int J Parasitol 1999; 29: 1241-1247.

11. Spano F, Putignani L, McLauchlin J, Casemore DP, Crisanti A. PCR-RFLP analysis of the Cryptosporidium oocyst wall protein (COWP) gene discriminates between $C$. wrairi and $C$. parvum, and between $C$. parvum isolates of human and animal origin.
FEMS Microbiol Lett 1997; 150: 209-217.

12. Spano F, Putignani L, Guida S, Crisanti A. Cryptosporidium parvum: PCR-RFLP analysis of the TRAP-C1 (thrombospondin-related adhesive protein of Cryptosporidium-1) gene discriminates between two alleles differentially associated with parasite isolates of animal and human origin. Exp Parasitol 1998; 90: 195-198.

13. Peng MM, Xiao L, Freeman AR et al. Genetic polymorphism among Cryptosporidium parvum isolates: evidence of two distinct human transmission cycles. Emerg Infect Dis 1997; 3: $567-573$.

14. Sulaiman IM, Morgan UM, Thompson RCA, Lal AA, Xiao L. Phylogenetic relationships of Cryptosporidum parasites based on the 70-kilodalton heat shock protein (HSP70) gene. Appl Environ Microbiol 2000; 66: 2385-2391.

15. Xiao L, Escalante L, Yan $\mathrm{C}$ et al. Phylogenetic analysis of Cryptosporidium parasites based on the small-subunit rRNA gene locus. Appl Environ Microbiol 1999; 65: 1578-1583.

16. Fayer R, Speer CA, Dubey JP. The general biology of Cryptosporidium. In: Fayer $\mathrm{R}$ (ed) Cryptosporidium and cryptosporidiosis. Boca Raton, FL, CRC Press. 1997: 1-41.

17. Morgan UM, Sargent KD, Elliot A, Thompson RCA. Cryptosporidium in cats: additional evidence for C. felis. Vet $J$ 1998; 156: 159-161.

18. Sargent KD, Morgan UM, Elliot A, Thompson RCA. Morphological and genetic characterisation of Cryptosporidium oocysts from domestic cats. Vet Parasitol 1998; 77: 221-227.

19. Morgan UM, Xiao L, Monis P et al. Cryptosporidium spp. in domestic dogs: the "dog" genotype. Appl Environ Microbiol 2000; 66: 2220-2223.

20. Rose JB, Lisle JT, LeChevallier M. Waterborne cryptosporidiosis: incidence, outbreaks, and treatment strategies. In: Fayer $\mathrm{R}$ (ed) Cryptosporidium and cryptosporidiosis. Boca Raton, FL, CRC Press. 1997: 93-109.

21. McLauchlin J, Pedraza-Díaz S, Amar C, Nichols GL. Molecular epidemiological analysis of Cryptosporidium spp. in the UK: fecal samples from humans and 105 fecal samples from livestock animals. J Clin Microbiol 2000; 38: 3984-3990. 